\title{
Adenomyosis a Rare Contributor to Severe Primary Post-Partum Haemorrhage
}

\author{
Ayokunle Moses Olumodeji ${ }^{* 1}$, Oluwabusayo Abayomi Aborisade ${ }^{\mathbf{2}}$, Ayodeji Kayode Adefemi ${ }^{\mathbf{1}}$, \\ Modupe Olatokunbo Adedeji ${ }^{1}$, Ufuoma Oluwaseyi Olumodeji ${ }^{3}$ \\ ${ }^{1}$ Department of Obstetrics and Gynaecology, Lagos State University Teaching Hospital, Lagos, Nigeria \\ ${ }^{2}$ Department of Obstetrics and Gynaecology, Island Maternity, Lagos, Nigeria \\ ${ }^{3}$ Department of Ophthalmology, Lagos State University Teaching Hospital, Lagos Nigeria
}

*Corresponding author: Ayokunle Moses Olumodeji; ayokunleolumodeji@yahoo.com

Received 16 April 2021;

Accepted 14 May 2021;

Published 26 May 2021

\begin{abstract}
Adenomyosis is a challenging clinical condition for women of reproductive age with both proven and speculated, major gynaecologic and obstetric consequences. We present the case of a woman who had adenomyosis of the uterus, a successful in-vitro fertilized (IVF) conceived twin pregnancy, and severe primary postpartum haemorrhage (PPH) during caesarean section. The 38 -year-old, gravida 2 , para $0^{+1}$, with a 6 -year history of prior infertility, had severe primary postpartum haemorrhage at caesarean delivery of a live set of twins at estimated gestational age of 35 weeks. Conservative treatment included uterine compression and massage, blood transfusion, administration of intravenous carbetocin and application of multiple figure of eight haemostatic sutures at the placenta bed. Intra-operative finding on gross inspection of the uterus was in keeping with focal adenomyosis in the region of the placenta bed. Biopsy of the involved myometrial wall was avoided due to risk of provoking further severe haemorrhage. Her prior medical record revealed pelvic endometriosis at diagnostic laparoscopy prior to conception while being evaluated for infertility. Women with prior infertility and/or endometriosis, due to very probable co-existing adenomyosis, are at high risk of life-threatening, severe post-partum haemorrhage at delivery.
\end{abstract}

Keywords: Adenomyosis, Adenomyosis in Pregnancy, Primary Postpartum Haemorrhage, Severe Postpartum Haemorrhage.

\section{Introduction}

Adenomyosis is a benign condition characterized by uterine enlargement due to the presence of endometrial stroma and glands within the myometrium ${ }^{[1]}$. Adenomyosis has a significant impact on women's lives and is typically associated with chronic pelvic pain, dysmenorrhea, heavy menstrual periods and infertility ${ }^{[1]}$. Definitive diagnosis of adenomyosis requires histological confirmation, however, since $30-40 \%$ of patients remain asymptomatic, the actual prevalence of the disease remains unknown ${ }^{[1,2]}$. The prevalence has been reported as ranging from $1 \%$ to $70 \%$ according to different studies, reflecting the lack of standard diagnostic criteria ${ }^{[2]}$.

The frequency of adenomyosis in pregnancy has been increasing in recent years, most likely due to the increase in the number of pregnancies in women of advanced age and increase in pregnancies achieved by fertility treatments ${ }^{[3]}$. Adenomyosis has been reported to be associated with poor pregnancy outcomes, including an increased risk of preterm delivery, preterm premature rupture of membranes (PPROM), and fetal growth restriction (FGR) ${ }^{[4]}$. However, the potential impact of adenomyosis on the maternal and foetal outcomes of pregnancy and its association with $\mathrm{PPH}$ is still unclear ${ }^{[1]}$.

Adenomyosis may increase the risk of PPH as a result of abnormal placental localization, as part of the placenta previa spectrum ${ }^{[1]}$. Equally, abnormal trophoblast invasion into the myometrial junctional zone could lead to several forms of accreta, therefore causing incomplete placental detachment or a retained placenta which generally lead to uterine atony and PPH ${ }^{[1,5]}$. Another important factor that could contribute to an increased risk of $\mathrm{PPH}$ is the characteristic neoangiogenesis specific to adenomyosis ${ }^{[6]}$. Studies on adenomyosis have demonstrated that myocytes are structurally different from those of normal uteri. These changes are thought to be responsible for a disturbance in normal calcium cycling with a consequent alteration of normal contractility ${ }^{[7]}$. This defective contractility could result in an incongruous myometrial contraction after placental expulsion and lead to $\mathrm{PPH}^{[1]}$.

Therefore, women with adenomyosis may require specific management during pregnancy and delivery to prevent severe PPH. We report a woman with IVF conceived twin gestation with coexisting adenomyosis who developed severe PPH. 


\section{Case Presentation}

A 38year old booked $\mathrm{G}_{2} \mathrm{P}_{0}{ }^{+1}$ (1 mid-trimester miscarriage) trader, with an IVF-conceived twin gestation, presented to the antenatal clinic at an estimated gestational age of 15 weeks and three days. She had a history of six years infertility secondary to endometriosis diagnosed at laparoscopy and had an IVF done a year prior to presentation, which resulted in a miscarriage at 19 weeks. In index pregnancy, she had a cervical cerclage done at estimated gestational age (EGA) of 17 weeks. She was a known hypertensive patient; with good blood pressure control on dietary and life-style modification and she was not on any antihypertensive.

The booking packed cell volume (PCV) was $33 \%$ with genotype AA and blood group $\mathrm{O}$ positive. She was screened for HIV, hepatitis and syphilis, all of which were negative. She had three obstetric scans done which revealed monochorionic diamniotic twin gestation with no abnormality detected. She was on routine ANC drugs. She was commenced on subcutaneous clexane, 40mg daily, in the 3rd trimester for thromboprophylaxis.

The antenatal period was largely uneventful until 35 weeks of pregnancy when she was noticed to have poor blood pressure control. She was admitted and had caesarean delivery on account of poor blood pressure control in a chronic hypertensive, with IVF conceived twin gestation at EGA of 35weeks.

At surgery, the abdominal cavity was accessed through a Joel Cohen incision and a normal looking lower uterine segment was found. A curvilinear incision was made on the lower uterine segment and a set of live twins were delivered with good APGAR scores. Placenta was delivered manually without difficulty and was complete with no missing cotyledons. There was no evidence of invasive placentation.

There was profuse bleeding following delivery of the placenta which was initially ascribed to atony. However, hemorrhage did not subside despite use of therapeutic dose of oxytocic (IV carbetocin 100ug stat) and an obviously well contracted uterus. Bleeding was identified from the right posterofundal area of the uterus. This area, which spanned about $7 \times 5 \mathrm{~cm}$, was found to be friable and riddled with yellowish-white, firm, patchy lesions, without clear-cut junction or capsule between the adjoining normal myometrium. This was suggestive of adenomyosis. Attempt at hysterectomy was aborted due to dense adhesions involving all the pelvic organs as well as the rectum and sigmoid colon. Biopsy of the suspected adenomyosis lesion was not taken due to possible worsening of the severe hemorrhage. Hemostasis was eventually secured with the use of hemostatic sutures (figure-of-eight) and intermittent repeated pressure on the bleeding sites as well as the placental beds. She was resuscitated with 3 units of whole blood intra-operatively and subsequently had
2 more units of whole blood and 2 units of fresh frozen plasma post-operatively. Estimated blood loss was 2.1L.

Following surgery, she was monitored at the intensive care unit. Post-operative recovery was good. She remained stable and was discharged home on the $7^{\text {th }}$ day post-op. Post-natal course was not adversely eventful. Post-partum magnetic resonance imaging wasn't done because she did not consent to it. Lack of biopsy specimen for histologic confirmation of uterine adenomyosis is a limitation of this report; however intra-op findings with the laparoscopic confirmation of endometriosis prior to conception suggest that the uterine lesion at the placental bed was most likely adenomyosis of the uterus.

\section{Conclusion}

As artificial reproductive techniques for infertile couples become more refined, widespread and successful, the number of pregnancies with co-existing endometriosis and/or adenomyosis will continue to rise. At delivery, women with adenomyosis in pregnancy should be managed as very high risk for severe primary post-partum haemorrhage.

\section{References}

[1] Martone S, Centini G, Exacoustos C, Zupi E, Afors K, Zullo F, et al. Pathophysiologic mechanisms by which adenomyosis predisposes to postpartum haemorrhage and other obstetric complications. Medical hypotheses. 2020;143:109833.

[2] Struble J, Reid S, Bedaiwy MA. Adenomyosis; a clinical review of a challenging gynecologic condition. J Minimally Invasive Gynecol 2015; 23(2):164-185.

[3] Kunz G, Herbertz M, Beil D, Huppert P, Leyendecker G. Adenomyosis as a disorder of the early and late human reproductive period. Reprod Biomed Online. 2007;15:681-685.

[4] Tamura H, Kishi H, Kitade M, et al. Complications and outcomes of pregnant women with adenomyosis in Japan. Reprod Med Biol. 2017;16(4):330-336.

[5] Yinka O, Candev VA. Postpartum Hemorrhage: Epidemiology, Risk Factors, and Causes. Clinical obstetrics and gynecology. 2010; 53(1):147-156.

[6] Weis SM, Cheresh DA. Pathophysiological consequences of VEGF-induced vascular permeability. Nature. 2005;437:497-504.

[7] Mehasseb MK, Bell SC, Pringle JH, Habiba MA. Uterine adenomyosis is associated with ultrastructural features of altered contractility in the inner myometrium. Fertility and Sterility. 2010;93(7):2130-6. 